\title{
Numeral Recognition Using Statistical Methods Comparison Study
}

\author{
Huda A. Rasheed *
}

\author{
Nada A. Rasheed **
}

\author{
Received 3, December, 2009 \\ Accepted 11, April, 2010
}

\begin{abstract}
:
The area of character recognition has received a considerable attention by researchers all over the world during the last three decades. However, this research explores best sets of feature extraction techniques and studies the accuracy of wellknown classifiers for Arabic numeral using the Statistical styles in two methods and making comparison study between them. First method Linear Discriminant function that is yield results with accuracy as high as $90 \%$ of original grouped cases correctly classified. In the second method, we proposed algorithm, The results show the efficiency of the proposed algorithms, where it is found to achieve recognition accuracy of $92.9 \%$ and $91.4 \%$. This is providing efficiency more than the first method.
\end{abstract}

Key words: Numeral, Recognition, Discriminant

\section{Introduction:}

Document image analysis systems can contribute tremendously to the advancement of the automation process and can improve the interaction between man and machine in many applications, including office automation, check verification and a large variety of banking, business and data entry applications [1].

Character recognition is a longstanding, fundamental problem in pattern recognition. It has been the subject of a considerable number of studies and serves many useful applications. Of the two major issues in character recognition, character shape representation and category assignment, classification has been, by and large, the subject of most studies. These studies assumed that descriptions of shape by basic characteristics such as curvature, tangents, and transform coefficients, are sufficiently expressive to justify focusing on classification. However, a good representation is as important as a good classifier for high performance [2].

There are two types of character recognition systems: on-line and offline systems. Each system has its own algorithms and methods. The main difference between them is that in an on-line system the recognition is performed in the time of writing while the off-line recognition is performed after the writing is completed [3].

The recognition of hand 4 written numeral characters has been a topic widely studied during the recent decades because of both its theoretical value in pattern recognition and its numerous possible applications [4], one such area is the reading of courtesy amounts on bank checks. This application has been very popular in handwriting recognition research, due to the availability of relatively inexpensive CPU power, and the possibility to reduce considerably the manual effort involved in this task. Another application is the reading of

*AL- Mustansiriyah University/ College of sciences/ Department of Mathematics.

**Babylon University /Computer Science . 
postal zip codes in addresses written or typed on envelopes. The former is more difficult than the latter due to a number of differences in the nature of the handwritten material. For example, bank checks systems [5].

Pattern recognition systems typically involve two steps: feature extraction in which appropriate representation of pattern are developed and classification in which decision rules for separating pattern classes are defined. There are indeed as many possible features as the ways characters are written. These features can be classified into two major categories: statistical and structural features. In the statistical approaches the input pattern is characterized by a set of $\mathrm{N}$ features and its description is achieved my means of a feature vector belonging to an $\mathrm{N}$-dimensional space. On the other hand, in structural approaches it is assumed that the pattern to be recognized can be decomposed into simpler components (called primitive) and then described in terms of simple appropriate attributes of primitives and their topological relations [6].

This paper is structured as the following: Section 2 provides a brief overview of the liner discernment functions, Section 3 presents the feature extraction from handwritten numeral, Section 4 describes the overall experiments and results and Section 5 includes some discussion and comparison. Finally, Section 6 presents our recommendations.

\section{Linear Discriminant Analysis (LDA)}

Linear Discriminant Analysis (LDA) is a method of finding such a linear combination of variables which best separates two or more classes. It aims at the classification of an object into one of $K$ given classes based on information from a set of $p$ predictor variables. Among the many available methods, the simplest and most popular approach is linear discriminant analysis (LDA)

Fisher Linear Discriminant Analysis means finds a linear transformation of predictor variables which provides a more accurate discrimination (see the right).

- Objective is to find $\mathrm{w}$ to maximize $\mathrm{J}(\mathrm{w})$, where:

$J(w)=\frac{w^{T} S_{B} w}{w^{T} S_{W} w}$

- $\mathrm{S}_{\mathrm{B}}$ is between class scatter matrixes, related to covariance matrix of centers of clusters

$$
S_{B}=\sum N_{c}\left(\mu_{c}-\bar{x}\right)\left(\mu_{c}-\bar{x}\right)^{T} \ldots(2)
$$

$-N c$ is the number of observations in class c.

$$
\begin{aligned}
& \mu_{c}=\frac{1}{N_{c}} \sum_{i \in c} x_{i} \\
& \bar{x}==\frac{1}{N} \sum_{i} x_{i}=\frac{1}{N} \sum_{c} N_{c} \mu_{c}
\end{aligned}
$$

- $S_{W}$ is within class scatter matrix, related to covariance matrix between clusters

$$
S_{W}=\sum_{c} \sum_{i \in c}\left(x_{i}-\mu_{c}\right)\left(x_{i}-\mu_{c}\right)^{T} \ldots .(3)
$$

Good Class Separation includes Finding the direction to project data on so that:

- Between classes variance is maximized.

- Within class Variance is minimized.

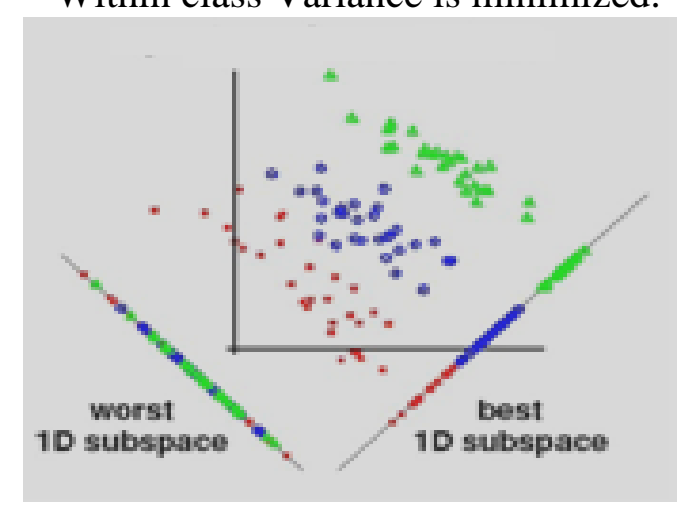

Fig(1): class feature data. 


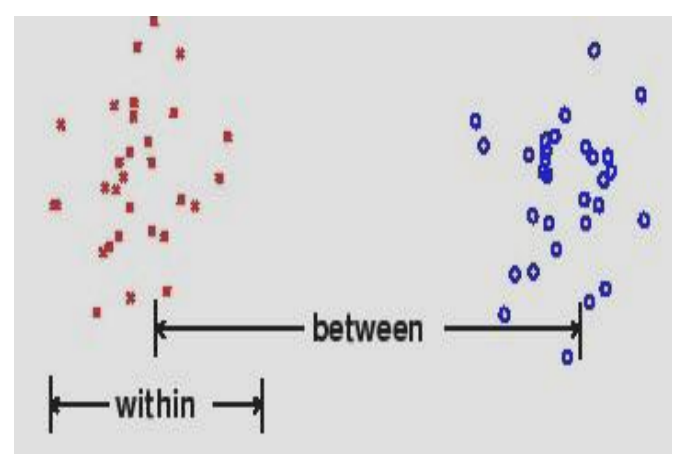

Fig.(2): Good class separation

\section{Feature Extraction}

The issue of how many features a method needs to use to obtain reliable handwritten numeral recognition is a very difficult one. There is always a temptation to include more and more features in a method in a hope of improving performance.

Most methods, but not all, during the feature extraction, would generate a reference numeral (or a set of reference numeral) for each individual. This normally requires a number of numeral of the user to be captured at enrollment or registration time (we call this numeral the sample numeral). When a user claims to be a particular individual and presents a numeral (we call this numeral the test numeral), the test numeral is compared with the reference numeral for that individual using one of the methods in this paper. To preparing the numeral database is to determine the number of sample numeral that need to be used in recognition, here the sample is (70) digits, that collected from different persons and used their numeral in database.

To reduce the variation in size, all numeral images will be scaled at fixed size $(77 \times 77)$ pixels, if the scanned numeral is large or smaller, then it will be resized so that all numerals are having the same size. After the images were acquired, they were converted into monochrome bitmap (BMP) form, it was necessary to convert the images into binary representations of the handwriting.

In the present case, the numeral images were divided into 7 segments where each segment consists of $1 \times 77$ pixels, as in Figure (3), each segments is represented features or measurements as vector (length 0.0 , length 0.2 , length 0.4 , length 0.5 , length 0.6 , length 0.8 , and length 1.0). In addition to the value of tall feature that is represented the length column between the points (a) and (b) as shown in figure (3) below, this feature is necessary to different between (4 and 9) numerals.

It is viewed as a point in a dimensional space $(8 \times 70)$, the goal is to choose those features that allow pattern vectors belonging to different categories.

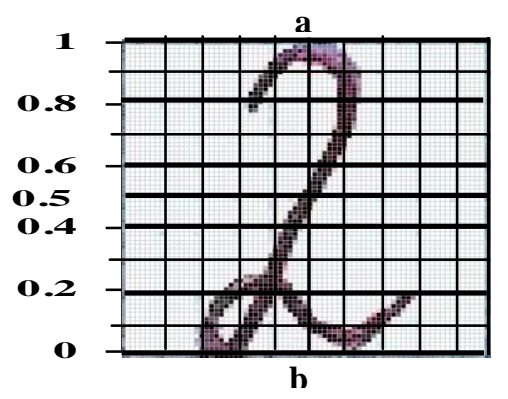

Fig.(3): Decomposition of a numeral (Features)

\section{Materials and Methods:}

After feature extraction stage begins, the result of this stage is a vector of (8) values for each numeral and there are (70) numeral for different persons in all database.

\section{Recognition using linear discriminant function}

The efficiency of the linear discrimination technique has been tested over a wide range of handwritten character recognition problems.

We had several features extracted from the handwritten numeral, the vector (length.0, length.2, length.4, length.5, length.6, length.8, and length1, tall) for each numeral which 
created matrix dimension $(8 \times 70)$. We tried LDA using SPSS.v11 software (Statistical Package for Social Sciences) and applied Fisher's linear discriminant functions then we get the Classification Function Coefficients, results as shown in the table (1) below:

Table (1): Classification Function Coefficients by using Fisher's linear discriminant functions

\begin{tabular}{|c|c|c|c|c|c|c|c|c|c|c|}
\hline \multirow{2}{*}{ LENGTH } & \multicolumn{10}{|c|}{ GROUP } \\
\hline & 0 & 1 & 2 & 3 & 4 & 5 & 6 & 7 & 8 & 9 \\
\hline V1 & .153 & .215 & .211 & .416 & .208 & .760 & .292 & 1.023 & .461 & .360 \\
\hline V.8 & .307 & -.014 & .334 & -.078 & .344 & -.248 & -.087 & $\begin{array}{l}-.073 \\
\end{array}$ & .155 & .305 \\
\hline V.6 & .088 & -.354 & -.447 & -.323 & .409 & -.312 & -.720 & -.619 & -.666 &.- .107 \\
\hline V.5 & .289 &.- .121 & $\begin{array}{l}-.003 \\
\end{array}$ & $\begin{array}{l}-.064 \\
\end{array}$ & .155 & $\begin{array}{l}-.016 \\
\end{array}$ & $\begin{array}{c}.003 \\
\end{array}$ & \begin{tabular}{|l|}
-.049 \\
\end{tabular} & .136 & $\begin{array}{l}-.075 \\
\end{array}$ \\
\hline V.4 & .514 & .205 & .041 & .197 & -.087 & .310 & .358 & .402 & .454 & .067 \\
\hline V.2 & .105 & $\begin{array}{l}-.450 \\
\end{array}$ & $\begin{array}{l}-.357 \\
\end{array}$ & $\begin{array}{l}-.379 \\
\end{array}$ & $\begin{array}{l}-.403 \\
\end{array}$ & $\begin{array}{l}.129 \\
\end{array}$ & .286 & $\begin{array}{l}-.421 \\
\end{array}$ & .230 & -.497 \\
\hline V.0 & .184 & -.026 & .220 & -.008 & -.034 & -.010 & .139 & -.287 & .122 & -.100 \\
\hline TALL & 3.536 & 3.996 & 3.928 & 3.954 & 1.756 & 3.525 & \begin{tabular}{|l|l|}
3.778 \\
\end{tabular} & 4.104 & 3.762 & 3.912 \\
\hline (Constant) & $\begin{array}{c}- \\
167.679 \\
\end{array}$ & $\begin{array}{c}- \\
154.851 \\
\end{array}$ & $\begin{array}{c}- \\
159.852 \\
\end{array}$ & $\begin{array}{c}- \\
154.126 \\
\end{array}$ & $\begin{array}{c}- \\
46.514 \\
\end{array}$ & $\begin{array}{c}- \\
133.942 \\
\end{array}$ & $\begin{array}{c}- \\
154.301 \\
\end{array}$ & $\begin{array}{c}- \\
178.802 \\
\end{array}$ & $\begin{array}{c}- \\
161.581\end{array}$ & $\begin{array}{c}- \\
156.848 \\
\end{array}$ \\
\hline
\end{tabular}

To recognize numeral it must calculate the vector (length.0, length.2, length.4, length.5, length.6, length.8, length1, tall) to the unknown number. Then redress them in group of ten functions that computed according in the table (1) above for each numeral as shown below then the unknown numeral belong to the class which consideration the equation that afford from largest value.

The following table (2) summarizes the main outputs of LDA, which gives a good classification of $90 \%$ of original grouped cases correctly classified.

\author{
$\operatorname{Group}(0)=-167.679+3.536($ TALL $)+.184$ \\ (LENGTH.0)+0.105 \\ (LENGTH.2)+0.514 \\ (LENGTH.4) + 0.289 \\ (LENGTH.5) + \\ 0.088(LENGTH.6) + \\ 0.307(LENGTH.8) + \\ 0.153(LENGTH1) \\ Group $(9)=-156.848+3.912($ TALL $)-.100$ \\ (LENGTH.0) - .497 \\ $($ LENGTH.2) +.067 \\ (LENGTH.4) - .075 \\ (LENGTH.5) - . .107 \\ (LENGTH.6) +.305 \\ (LENGTH.8) +.360 \\ (LENGTH1)
}

Table (2): Classification Results according to Fisher Linear Discriminant

\begin{tabular}{|c|c|c|c|c|c|c|c|c|c|c|c|c|}
\hline \multirow{2}{*}{\multicolumn{2}{|c|}{ GROUP }} & \multicolumn{10}{|c|}{ Predicted Group Membership } & \multirow{2}{*}{ Total } \\
\hline & & $\mathbf{0}$ & 1 & 2 & 3 & 4 & 5 & 6 & 7 & 8 & 9 & \\
\hline \multirow{10}{*}{ Count } & 0 & 7 & $\mathbf{0}$ & $\mathbf{0}$ & $\mathbf{0}$ & $\mathbf{0}$ & $\mathbf{0}$ & $\mathbf{0}$ & $\mathbf{0}$ & $\mathbf{0}$ & $\mathbf{0}$ & 7 \\
\hline & 1 & $\mathbf{0}$ & 7 & 1 & $\mathbf{0}$ & $\mathbf{0}$ & $\mathbf{0}$ & $\mathbf{0}$ & $\mathbf{0}$ & $\mathbf{0}$ & $\mathbf{0}$ & 8 \\
\hline & 2 & $\mathbf{0}$ & $\mathbf{0}$ & 7 & $\mathbf{0}$ & $\mathbf{0}$ & $\mathbf{0}$ & $\mathbf{0}$ & $\mathbf{0}$ & $\mathbf{0}$ & $\mathbf{0}$ & 7 \\
\hline & 3 & $\mathbf{0}$ & $\mathbf{0}$ & $\mathbf{0}$ & 6 & $\mathbf{0}$ & $\mathbf{0}$ & 1 & $\mathbf{0}$ & $\mathbf{0}$ & $\mathbf{0}$ & 7 \\
\hline & 4 & $\mathbf{0}$ & $\mathbf{0}$ & $\mathbf{0}$ & $\mathbf{0}$ & 8 & 0 & 0 & 0 & $\mathbf{0}$ & 0 & 8 \\
\hline & 5 & $\mathbf{0}$ & $\mathbf{0}$ & $\mathbf{0}$ & 1 & $\mathbf{0}$ & 3 & 1 & $\mathbf{0}$ & $\mathbf{0}$ & $\mathbf{0}$ & 5 \\
\hline & 6 & $\mathbf{0}$ & $\mathbf{0}$ & $\mathbf{0}$ & $\mathbf{0}$ & $\mathbf{0}$ & $\mathbf{0}$ & 6 & $\mathbf{0}$ & 2 & 0 & 8 \\
\hline & 7 & $\mathbf{0}$ & $\mathbf{0}$ & $\mathbf{0}$ & $\mathbf{0}$ & $\mathbf{0}$ & $\mathbf{0}$ & $\mathbf{0}$ & 6 & 0 & 0 & 6 \\
\hline & 8 & $\mathbf{0}$ & $\mathbf{0}$ & $\mathbf{0}$ & $\mathbf{0}$ & $\mathbf{0}$ & $\mathbf{0}$ & $\mathbf{0}$ & $\mathbf{0}$ & 7 & $\mathbf{0}$ & 7 \\
\hline & 9 & $\mathbf{0}$ & 1 & $\mathbf{0}$ & $\mathbf{0}$ & $\mathbf{0}$ & $\mathbf{0}$ & $\mathbf{0}$ & $\mathbf{0}$ & $\mathbf{0}$ & 6 & 7 \\
\hline \multirow{10}{*}{$\%$} & 0 & 100.0 & .0 & .0 & .0 & .0 & .0 & .0 & .0 & .0 & .0 & 100.0 \\
\hline & 1 & .0 & 87.5 & 12.5 & .0 & .0 & .0 & .0 & .0 & .0 & .0 & 100.0 \\
\hline & 2 & .0 & .0 & 100.0 & .0 & .0 & .0 & .0 & .0 & .0 & .0 & 100.0 \\
\hline & 3 & .0 & .0 & .0 & 85.7 & .0 & .0 & 14.3 & .0 & .0 & .0 & 100.0 \\
\hline & 4 & .0 & .0 & .0 & .0 & 100.0 & .0 & .0 & .0 & .0 & .0 & 100.0 \\
\hline & 5 & .0 & .0 & .0 & 20.0 & .0 & $\begin{array}{l}60.0 \\
\end{array}$ & 20.0 & .0 & .0 & .0 & 100.0 \\
\hline & 6 & .0 & .0 & .0 & .0 & .0 & .0 & 75.0 & .0 & 25.0 & .0 & 100.0 \\
\hline & 7 & .0 & .0 & .0 & .0 & .0 & .0 & .0 & 100.0 & .0 & .0 & 100.0 \\
\hline & 8 & .0 & .0 & .0 & .0 & .0 & .0 & .0 & .0 & 100.0 & .0 & 100.0 \\
\hline & 9 & .0 & \begin{tabular}{|l|l|}
14.3 \\
\end{tabular} & .0 & .0 & .0 & .0 & .0 & .0 & .0 & 85.7 & 100.0 \\
\hline
\end{tabular}


This presents successfully features, which are extraction and efficiently the method Fisher Linear Discriminant Analysis in recognize numeral.

\section{Recognition using the proposed algorithm}

After extracting feature vectors having (8) elements for each numeral available as the database, these vectors will represent the input to the numeral pattern recognizer. Then we are applying the proposed algorithm that can be presented as follows:

\section{The proposed algorithm is shown as follows:}

Step1: For $\mathrm{i}=0$ to 9

Step2: For $\mathrm{j}=1$ to $\mathrm{n}_{\mathrm{i}},\left(\mathrm{n}_{\mathrm{i}}\right.$ represented the number of replication of the class $\mathrm{i}$, $\mathrm{i}=0,1,2, \ldots, 9)$

Step3: Input features (Length.0,Length.2, Length.4, Length.5, Length.6, Length.8, Length1, Tall)

\section{Step3.1: Next $\mathrm{j}$}

Step4: Depending on $\mathrm{n}_{\mathrm{i}}$ values, calculate average to each feature: $\overline{\text { Length } 0} i, \overline{\text { Length } 2} i, \ldots . ., \overline{\mathrm{T} \text { all }}$

through class i, as a result it will be a vector of order 8 .

Step5: Depending on $n_{i}$ valus, calculate standard deviation to each feature:

$\mathrm{S}_{0 \mathrm{i}}, \mathrm{S}_{.2 \mathrm{i}}, \mathrm{S}_{.4 \mathrm{i}}, \mathrm{S}_{.5 \mathrm{i}}, \mathrm{S}_{.6 \mathrm{i}}, \mathrm{S}_{.8 \mathrm{i}}, \mathrm{S}_{1 \mathrm{i}}$ through class $\mathrm{i}$, as a result it will be a vector of order 8 .

\section{Step5.1: Next i}

Step6: For $\mathrm{i}=0$ to 9

Step7: Substitute features for unknown numeric (v0, v0.2, v0.4, v0.5, v0.6, v0.8, v1, v.Tall ) into

the following equation:

$$
\begin{aligned}
& d_{i}=\frac{|v 0-\overline{\text { Length. } 0} i|}{S_{o i}}+\frac{|v 0.2-\overline{\text { Length. } 2} i|}{S_{2 i}}+\frac{|v 0.4-\overline{\text { Length. } 4} i|}{S_{4 i}}+\frac{|v 0.5-\overline{\text { Length. } 5 i}|}{S_{5 i}} \\
& +\frac{\mid v 0.6-\overline{\text { Length. }} \boldsymbol{i} i}{\mid}+\frac{|v 0.8-\overline{\text { Length.8 }}|}{S_{6 i}}+\frac{|v 1-\overline{\text { Length.1 }}|}{S_{8 i}}+\mid v . \text { Tall- } \overline{\text { Talli }} \mid
\end{aligned}
$$

(4)

Where $\mathrm{i}$ represents the class $(i=0,1,2, \ldots, 9)$.

Step7.1: Next i

Step8: Classifying unknown number into class which corresponds to Minimum value of $d_{i}$

Firstly, the data used in recognition involves of the vectors values (Length.0, Length.2, Length.4, Length.5, Length.6, Length.8,
Length1, Tall) average each features through each class to get the table (3) below: 
Table (3): The Distribution of Features Averages According to its class

\begin{tabular}{|c|c|c|c|c|c|c|c|c|}
\hline Class & Length1 & Length.8 & Length.6 & Length.5 & Length.4 & Length.2 & Length.0 & Tall \\
\hline $\mathbf{0}$ & 15.43 & 35.43 & 41.57 & 42.43 & 43.00 & 39.00 & 16.86 & 77.00 \\
\hline $\mathbf{1}$ & 3.75 & 7.63 & 3.88 & 4.00 & 4.13 & 4.38 & 12.13 & 77.00 \\
\hline $\mathbf{2}$ & 10.00 & 25.57 & 3.43 & 3.71 & 4.14 & 6.00 & 25.14 & 77.00 \\
\hline $\mathbf{3}$ & 13.43 & 6.57 & 9.00 & 9.14 & 4.57 & 8.43 & 13.71 & 77.00 \\
\hline $\mathbf{4}$ & 18.13 & 25.00 & 26.75 & 20.13 & 7.63 & 4.88 & 3.88 & 36.88 \\
\hline $\mathbf{5}$ & 29.20 & 4.00 & 16.40 & 15.40 & 10.40 & 18.60 & 13.40 & 70.00 \\
\hline $\mathbf{6}$ & 6.50 & 7.88 & 6.25 & 7.63 & 12.63 & 33.38 & 16.38 & 77.00 \\
\hline $\mathbf{7}$ & 38.83 & 18.67 & 5.83 & 12.33 & 15.67 & 5.17 & 4.33 & 77.00 \\
\hline $\mathbf{8}$ & 19.57 & 26.86 & 13.43 & 19.43 & 27.29 & 32.57 & 17.29 & 77.00 \\
\hline $\mathbf{9}$ & 17.86 & 29.00 & 16.57 & 4.86 & 5.14 & 9.86 & 7.43 & 77.00 \\
\hline
\end{tabular}

Also, the standard deviations to each feature through classes $0,1, \ldots, 9$ are shown in following table:

Table (4): The Distribution of Features Standard deviations According to its class

\begin{tabular}{|c|c|c|c|c|c|c|c|c|}
\hline Class & Length1 & Length.8 & Length.6 & Length.5 & Length.4 & Length.2 & Length.0 & Tall \\
\hline $\mathbf{0}$ & 7.96 & 6.02 & 6.13 & 6.80 & 6.86 & 6.30 & 7.73 & .00 \\
\hline $\mathbf{1}$ & 1.04 & 4.69 & .64 & .76 & .99 & .92 & 9.34 & .00 \\
\hline $\mathbf{2}$ & 3.11 & 7.50 & .53 & .76 & .38 & 4.55 & 13.48 & .00 \\
\hline $\mathbf{3}$ & 4.31 & 7.30 & 5.72 & 6.15 & 1.40 & 8.75 & 9.98 & .00 \\
\hline $\mathbf{4}$ & 12.46 & 6.05 & 5.60 & 14.12 & 7.93 & 1.64 & 1.36 & 6.38 \\
\hline $\mathbf{5}$ & 9.42 & 1.22 & 12.97 & 14.26 & 14.33 & 19.59 & 9.29 & 15.65 \\
\hline $\mathbf{6}$ & 4.78 & 9.82 & 6.43 & 10.31 & 13.86 & 6.82 & 6.67 & .00 \\
\hline $\mathbf{7}$ & 4.45 & 16.17 & .98 & 14.61 & 9.85 & 1.47 & .52 & .00 \\
\hline $\mathbf{8}$ & 7.55 & 9.17 & 3.87 & 5.97 & 5.88 & 4.31 & 5.53 & .00 \\
\hline $\mathbf{9}$ & 7.99 & 4.76 & 12.08 & 1.68 & 2.54 & 10.33 & 2.94 & .00 \\
\hline
\end{tabular}

Now, using table (3) and (4), we can classify any unknown numeral into its class easily by applying equation (4) depending on its features.
Experimental results are then presented and analyzed $92.9 \%$ of original grouped cases correctly classified.

Table (5): Classification Results according to proposed method

\begin{tabular}{|c|c|c|c|c|c|c|c|c|c|c|c|c|}
\hline \multirow{2}{*}{\multicolumn{2}{|c|}{ GROUP }} & \multicolumn{10}{|c|}{ Predicted Group Membership } & \multirow{2}{*}{ Total } \\
\hline & & \multirow{2}{*}{$\begin{array}{l}\mathbf{0} \\
7\end{array}$} & \multirow{2}{*}{$\begin{array}{l}\mathbf{1} \\
0\end{array}$} & \multirow{2}{*}{$\begin{array}{l}\mathbf{2} \\
0\end{array}$} & \multirow{2}{*}{$\begin{array}{l}3 \\
0\end{array}$} & \multirow{2}{*}{$\begin{array}{l}4 \\
0\end{array}$} & \multirow{2}{*}{$\begin{array}{l}\mathbf{5} \\
0\end{array}$} & \multirow{2}{*}{$\begin{array}{l}6 \\
0\end{array}$} & \multirow{2}{*}{$\begin{array}{l}7 \\
0 \\
\end{array}$} & \multirow{2}{*}{$\begin{array}{l}8 \\
0\end{array}$} & \multirow{2}{*}{$\begin{array}{l}9 \\
0\end{array}$} & \\
\hline Count & 0 & & & & & & & & & & & 7 \\
\hline & 1 & 0 & 7 & 0 & 1 & 0 & 0 & 0 & 0 & 0 & 0 & 8 \\
\hline & 2 & 0 & 0 & 5 & 1 & 0 & 0 & 0 & 0 & 0 & 1 & 7 \\
\hline & 3 & 0 & 0 & 0 & 6 & 0 & 0 & 0 & 0 & 0 & 1 & 7 \\
\hline & 4 & 0 & 0 & 0 & 0 & 8 & 0 & 0 & 0 & 0 & 0 & 8 \\
\hline & 5 & 0 & 0 & 0 & 0 & 0 & 4 & 1 & 0 & 0 & 0 & 5 \\
\hline & 6 & 0 & 0 & 0 & 0 & 0 & 0 & 8 & 0 & 0 & 0 & 8 \\
\hline & 7 & 0 & 0 & 0 & 0 & 0 & 0 & 0 & 6 & 0 & 0 & 6 \\
\hline & 8 & 0 & 0 & 0 & 0 & 0 & 0 & 0 & 0 & 7 & 0 & 7 \\
\hline & 9 & 0 & 0 & 0 & 0 & 0 & 0 & 0 & 0 & 0 & 7 & 7 \\
\hline \multirow{10}{*}{$\%$} & 0 & 100 & 0 & 0 & 0 & 0 & 0 & 0 & 0 & 0 & 0 & 100 \\
\hline & 1 & 0 & 87.5 & 0 & 12.5 & 0 & 0 & 0 & 0 & 0 & 0 & 100 \\
\hline & 2 & 0 & 0 & 71.4 & 14.3 & 0 & 0 & 0 & 0 & 0 & 14.3 & 100 \\
\hline & 3 & 0 & 0 & 0 & 85.7 & 0 & 0 & 0 & 0 & 0 & 14.3 & 100 \\
\hline & 4 & 0 & 0 & 0 & 0 & 100 & 0 & 0 & 0 & 0 & 0 & 100 \\
\hline & 5 & 0 & 0 & 0 & 0 & 0 & 80 & 20 & 0 & 0 & 0 & 100 \\
\hline & 6 & 0 & 0 & 0 & 0 & 0 & 0 & 100 & 0 & 0 & 0 & 100 \\
\hline & 7 & 0 & 0 & 0 & 0 & 0 & 0 & 0 & 100 & 0 & 0 & 100 \\
\hline & 8 & 0 & 0 & 0 & 0 & 0 & 0 & 0 & 0 & 100 & 0 & 100 \\
\hline & 9 & 0 & 0 & 0 & 0 & 0 & 0 & 0 & 0 & 0 & 100 & 100 \\
\hline
\end{tabular}


Example (1): Suppose that the features of unknown number where as follows:

\begin{tabular}{|c|c|c|c|c|c|c|c|}
\hline Length1 & Length.8 & Length.6 & Length.5 & Length.4 & Length.2 & Length.0 & Tall \\
\hline 40 & 6 & 35 & 5 & 4 & 42 & 14 & 77 \\
\hline
\end{tabular}

Depending on means and Standard deviations of features for each class-as showing in tables 3,4 respectively- we applied Equation (4) and got these results:

\begin{tabular}{|c|c|c|c|c|c|c|c|c|c|c|}
\hline class & $\mathbf{0}$ & $\mathbf{1}$ & $\mathbf{2}$ & $\mathbf{3}$ & $\mathbf{4}$ & $\mathbf{5}$ & $\mathbf{6}$ & $\mathbf{7}$ & $\mathbf{8}$ & $\mathbf{9}$ \\
\hline di & 21.08 & 126.37 & 82.63 & 15.73 & 58.03 & 10.15 & 14.17 & 76.15 & 19.72 & 15.01 \\
\hline
\end{tabular}

Now, we can classify the unknown number into class 5 because the minimum value of di was 10.15 which correspond to class 5 .

There is another proposed algorithm which is the same as the previous algorithm except replacing equation (4) in step 7 by the following equation:

$$
\begin{aligned}
& d_{i}=\frac{(\mathrm{v} 0-\overline{\text { Length } 0} i)^{2}}{S_{o i}}+\frac{(\mathrm{v} 0.2-\overline{\text { Length } 2 i})^{2}}{S_{2 i}}+ \\
& \frac{(\mathrm{v} 0.4-\overline{\text { Length } 4 i})^{2}}{S_{4 i}}+\frac{(\mathrm{v} 0.5-\overline{\text { Length } 5 i})^{2}}{S_{5 i}}+ \\
& \frac{(\mathrm{v} 0.6-\overline{\text { Length } 6} i)^{2}}{S_{6 i}}+\frac{(\mathrm{v} 0.8-\overline{\text { Length } 8} i)^{2}}{S_{8 i}}+ \\
& \frac{(\mathrm{v} 1-\overline{\text { Length } 1 i})^{2}}{S_{1 i}}+\mid \mathrm{v} . \text { T all- } \overline{\text { Tall } i} \mid
\end{aligned}
$$

91.4\% of original grouped cases correctly classified by using this method, as shown in table (6):

Table (6): Classification Results according to second proposed method

\begin{tabular}{|c|c|c|c|c|c|c|c|c|c|c|c|c|}
\hline \multirow{2}{*}{ GROUP } & \multicolumn{10}{|c|}{ Tredicted Group Membership } \\
\cline { 2 - 12 } & & $\mathbf{0}$ & $\mathbf{1}$ & $\mathbf{2}$ & $\mathbf{3}$ & $\mathbf{4}$ & $\mathbf{5}$ & $\mathbf{6}$ & $\mathbf{7}$ & $\mathbf{8}$ & $\mathbf{9}$ & \\
\hline \multirow{6}{*}{ Count } & 0 & 7 & 0 & 0 & 0 & 0 & 0 & 0 & 0 & 0 & 0 & 7 \\
& 1 & 0 & 7 & 1 & 0 & 0 & 0 & 0 & 0 & 0 & 0 & 8 \\
& 2 & 0 & 0 & 7 & 0 & 0 & 0 & 0 & 0 & 0 & 0 & 7 \\
& 3 & 0 & 0 & 0 & 6 & 0 & 0 & 0 & 0 & 0 & 1 & 7 \\
& 4 & 0 & 0 & 0 & 0 & 6 & 0 & 0 & 0 & 0 & 2 & 8 \\
& 5 & 0 & 0 & 0 & 0 & 0 & 5 & 0 & 0 & 0 & 0 & 5 \\
& 6 & 0 & 0 & 0 & 0 & 0 & 0 & 7 & 0 & 1 & 0 & 8 \\
& 7 & 0 & 0 & 0 & 0 & 0 & 0 & 0 & 6 & 0 & 0 & 6 \\
& 8 & 0 & 0 & 0 & 0 & 0 & 0 & 0 & 0 & 7 & 0 & 7 \\
& 9 & 0 & 0 & 2 & 0 & 0 & 0 & 0 & 0 & 0 & 5 & 7 \\
\hline \multirow{6}{*}{$\%$} & 0 & 100.0 & 0. & 0. & 0. & 0. & 0. & 0. & 0. & 0. & 0. & 100.0 \\
& 1 & 0. & 87.5 & 12.5 & 0. & 0. & 0. & 0. & 0. & 0. & 0. & 100.0 \\
& 2 & 0. & 0. & 100.0 & 0. & 0. & 0. & 0. & 0. & 0. & 0. & 100.0 \\
& 3 & 0. & 0. & 0. & 85.7 & 0. & 0. & 14.3 & 0. & 0. & 0. & 100.0 \\
& 4 & 0. & 0. & 0. & 0. & 87.5 & 0. & 0. & 0. & 0. & 12.5 & 100.0 \\
& 5 & 0. & 0. & 0. & 0. & 0. & 80.0 & 20.0 & 0. & 0. & 0. & 100.0 \\
& 6 & 0. & 0. & 0. & 0. & 0. & 0. & 87.5 & 0. & 12.5 & 0. & 100.0 \\
& 7 & 0. & 0. & 0. & 0. & 0. & 0. & 0. & 100.0 & 0. & 0. & 100.0 \\
& 8 & 0. & 0. & 0. & 0. & 0. & 0. & 0. & 0. & 100.0 & 0. & 100.0 \\
& 9 & 0. & 14.3 & 0. & 0. & 0. & 0. & 0. & 0. & 0. & 85.7 & 100.0 \\
\hline
\end{tabular}

Example (2): Suppose that the features of unknown number as in example(1), to classify unknown number into its group using the second proposed method:

Apply Equation (5) and get these results: 
Now, we can classify the unknown number into class 5 because the minimum value of di was 87.8 which correspond to class 5 .

\section{Results and Discussion:}

The main conclusions of this paper can be summarized as follows: From table (5), we can see $(92.9 \%)$ of original grouped cases correctly classified by using the first proposed method, the second proposed method classified (91.4) of original grouped correctly, while the recognition using Fisher's linear discriminant functions method (using SPSS.V11) decreased into $(90 \%)$ of original grouped cases correctly classified, as a result, we can arrange compared method according to its efficiency as follows:

1- The first proposed method $(92.9 \%)$.

2- The second proposed method (91.4\%).

3- Fisher's linear discriminant functions method (90\%).

This namely the proposed algorithm can be depended in recognition the numerical.

\section{Recommendations}

The following recommendations for further research can be identified:

1- Employed the first proposed algorithm in pattern recognition and attempted the equation that be used in recognition (4) in form that increase the efficiency and accuracy the recognition.

2- Attempted to drawing the number as curves and fineding the equation to each numerical by used the partial derivation to be for Future Research.

\section{References:}

1-Mostafa G. M., 2004,"An Adaptive Algorithm for the Automatic Segmentation of Printed Arabic
Text", $17^{\text {th }}$ The national conference for computer. Abu-al-Aziz king University. Arabia Saudi.:437-444.

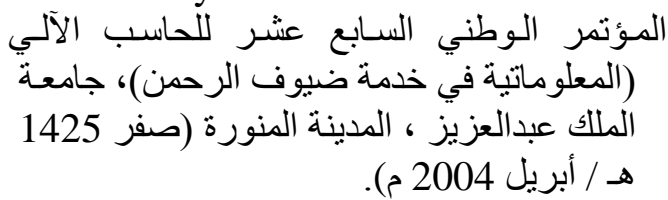

2- Ehsan N., N. Mezghani, A. Mitiche and R. de B. Johnston, 2006,"Online Persian/Arabic character recognition by polynomial representation and a Kohonen network", In Proceedings of the $18^{\text {th }}$ International Conference on Pattern Recognition (ICPR 2006), 4: 683-686, Hong Kong, China, August

3- Ahmed M. Z. and M. S. Zakaria, 2004,"Challenges in Recognizing Arabic Characters", $17^{\text {th }}$ the international conference for computer. Abu-al-Aziz king University. Arabia Saudi.:445-452.

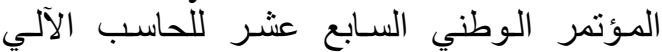

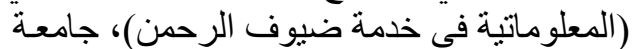

$$
\begin{aligned}
& \text { الملك عبدالعزيز ، المدينة المنورة (صفر } 1425 \\
& \text { هـ / أبريل } 2004 \text { م). }
\end{aligned}
$$

4- Suzete E. N. C , J. M. de Carvalho and R. Sabourin, 2002,"On the Performance of Wavelets for Handwritten Numerals Recognition", ICPR,Proceedings of the 16 th International Conference on Pattern Recognition (ICPR'02) ,3: 127-130.

5- Luiz S. O., R. Sabourin, F. vio Bortolozzi and C. Y. Suen, 2002,"Automatic Recognition of Handwritten Numeral Strings: A Recognition and Verification Strategy", IEEE Transactions On Pattern Analysis And Machine Intelligence,24(11):1438-1454.

6- Saeed M., Karim F. and Majid Z., 2005,"A Hybrid Structural/Statistical Classifier for Handwritten Farsi/Arabic Numeral Recognition", In IAPR Conference on Machine Vision Applications (MVA), May 16-18, 2005 Tsukuba Science City, Japan, ISBN 4-901122-04-5 S.64:218-221. 


\section{تمييز الأرقام باستخدام الطرق الإحصائية دراسة مقارنة}

ندى عبدالله رشيد**

$$
\text { هدى عبدالله رشبي" }
$$

|*الجامعة المستتصرية / كلية العلوم/ قسم الرياضيات

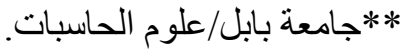

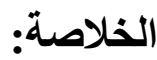

مجال تمييز الأحرف يلقى عناية واسعة من قبل الباحثين في اكثر العالم خلال الثثلاث العقود الأخيرة .

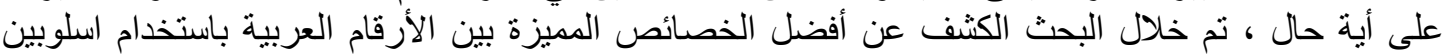
إحصائيين ومقارنة الدر اسة بينهم.

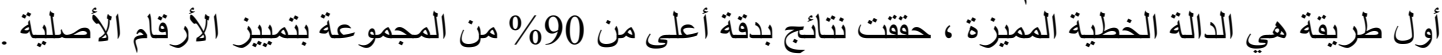

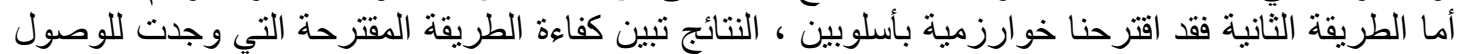
إلى تمييز بدقة 92.9\% و 91.4\% و هذه تقدم كفاءة أكثر من الطريقة الأولى. 